\title{
El papel de la descripción en la investigación cualitativa
}

\author{
THE ROLE OF DESCRIPTION IN QUALITATIVE RESEARCH
}

Mg. Juan Carlos Aguirre (jcaguirre@unicauca.edu.co) Departamento de Filosofía, Facultad de Ciencias Humanas y Sociales, Universidad del Cauca (Popayán, Colombia)

Dr. Luis Guillermo Jaramillo (ljaramillo@unicauca.edu.co) Departamento de Educación Física, Facultad de Ciencias Naturales, Exactas y de la Educación (Popayán, Colombia)

\begin{abstract}
In this paper we analyse the role of description in qualitative research. Our point is this: the description itself has a necessary role in qualitative research; though that role is necessary, it is not enough, interpretation is also necessary, but this interpretation must be supported on a realist epistemology setting up clearly what is observation. In order to defend this point we show some problems in the relationship description-interpretation; then, we choose two proposals that defend the role of description in qualitative research; finally, we respond what is the role of description in qualitative research.
\end{abstract}

Keywords: Description, Interpretation, Observation, Qualitative Research, Theory-Laden

\section{Resumen}

En este artículo analizamos cuál es el papel de la descripción en la investigación cualitativa. Afirmamos que la descripción en sí misma tiene un papel necesario en la investigación cualitativa; aunque este papel es necesario, no es suficiente, también se necesita la interpretación, pero esta interpretación debe respaldarse en una epistemología realista que tenga claro qué cuenta como observación. Para defender esta afirmación, mostramos algunos problemas en la relación descripción-interpretación; luego, elegimos dos propuestas que defienden el papel de la descripción en la investigación cualitativa y, finalmente, respondemos cuál es el papel de la descripción en la investigación cualitativa.

Palabras claves: descripción, interpretación, observación, investigación cualitativa, carga teórica.

\section{Introducción}

Durante muchas décadas las ciencias humanas y sociales tuvieron que lidiar con el debate en torno a si el tipo de investigación que adelantaban debía corresponderse con el modelo cuantitativo, esto es, con la investigación empírica y sistemática de fenómenos observables a partir de técnicas estadísticas, matemáticas o computacionales, o si más bien, debía ajustarse a los modelos cualitativos que, pese a la dificultad de una definición unívoca, podrían tomarse, de modo laxo, como aquellos que se dirigen a comprender de manera profunda la conducta humana y/o social y las razones que mueven tal conducta. Hoy, al parecer, las disputas han mermado su efusividad y hay cierto consenso en la posibilidad de combinar métodos cuantitativos y cualitativos en la investigación de las ciencias humanas y sociales, más 
aún, se ha ido consolidando el modelo de métodos mixtos (mixed-research o mixed methods research) como "el tercer mayor enfoque de investigación o paradigma de investigación" (Johnson, Onwuegbuzie y Turner 2007:112).

No obstante, el campo de las ciencias humanas y sociales continúa planteando desafíos interesantes para la epistemología. Los profesores Amedeo Giorgi y David L. Rennie parecen haber reactivado un debate acerca del papel de la descripción en la investigación cualitativa, específicamente, en investigación cualitativa en psicología, debate que exige clarificar algunos de los términos en discusión. Para Rennie la investigación cualitativa ha estado al margen de las corrientes principales de la psicología; su diagnóstico es que esta marginalización se debe, básicamente, a la "falta de una metodología coherente y unificada de los métodos de investigación cualitativa que aclare su credibilidad" (2012:385). Se infiere que su propósito es establecer unos principios que logren tal coherencia y unidad. En efecto, Rennie plantea cuatro proposiciones que sostendrían las metodologías cualitativas. No interesa en este artículo desplegar tales proposiciones; por ello, nos centramos solamente en la primera que es la que nutre la discusión que queremos plantear. Según Rennie, "la investigación cualitativa es hermenéutica, lo que implica la aplicación del método del círculo hermenéutico al texto acerca de la experiencia y/o acción" (2012:385), y más adelante: "la investigación cualitativa es metódicamente hermenéutica" (2012:388).

Por su parte, Giorgi afirma, replicando a Rennie, que pretende "demostrar que el método que h[a] desarrollado, basado en la fenomenología husserliana, no se puede interpretar tal y como Rennie se propone. Afirm[a] que, en general, una investigación psicológica puede considerarse interpretativa; sin embargo, cuando se emplea el método fenomenológico basado en Husserl, tal investigación es descriptiva" (2014:542). Uno de los aportes destacables del artículo de Giorgi consiste en que, a diferencia de algunas discusiones sobre el asunto, este define de manera precisa los términos en discusión, es decir, "descripción" e "interpretación", dándoles un lugar específico dentro del método fenomenológico que Giorgi ha propuesto a lo largo de su vida académica.

Insistimos que nuestro interés no es reconstruir las líneas finas de este debate, el cual tiene vetas prolíficas para varios asuntos (por ejemplo, para la fenomenología como método de investigación empírica), sino más bien recoger la discusión que le subyace: la distinción que pretende establecerse entre descripción e interpretación en la investigación cualitativa: ¿Toda investigación cualitativa es interpretativa? Si es así, ¿cumplen algún papel las descripciones? ¿Es posible integrar descripción e interpretación en las investigaciones en ciencias humanas y sociales? Ahora bien, estas preguntas específicas tienen relación con preguntas más amplias de la epistemología, por ejemplo, si es posible una observación libre de teoría, si la descripción garantiza la objetividad, si la interpretación está condenada a la subjetividad (en alusión al dictum Nietzscheano que no hay hechos sino solo interpretaciones) y, más allá de esto, si es posible una hermenéutica realista en ciencias humanas y sociales. El orden de consideración de estas preguntas será el siguiente: en el primer apartado trataremos de examinar la dupla descripción-interpretación bajo la dupla observación-teoría, sosteniendo que la observación/descripción no garantizan prima facie la objetividad, así como la interpretación/teoría no se comprometen necesariamente con la subjetividad. En el segundo apartado abordaremos lo que se conoce como descripción cualitativa (Sandelowski 2000), así como la hermenéutica realista (Ferraris 2012 y 2014), con el fin de mostrar propuestas que o bien dan un carácter propio a la descripción en investigación cualitativa, liberándola del lugar ancilar que parece haberle dado la tradición, o bien proponen una interpretación respaldada en el realismo, lo que implicará dar un peso a la observación/descripción. Finalmente, trataremos de dar respuesta a la pregunta implícita del título: ¿cuál es el papel de la descripción en la investigación cualitativa? Defenderemos que la sola descripción tiene un papel necesario e innegable en la investigación cualitativa; sin embargo, aunque tal 
papel es necesario, no es suficiente, se requiere de la interpretación, solo que esta tendrá que sostenerse en una epistemología realista, la cual, ineludiblemente, deberá tener claro qué cuenta como observación.

\section{Descripción-interpretación / observación-teoría}

Las tres primeras acepciones dadas por el Diccionario de la Real Academia Española (DRAE) del verbo 'describir' son: 1. "Delinear, dibujar, figurar algo, representándolo de modo que dé cabal idea de ello"; 2. "Representar a alguien o algo por medio del lenguaje, refiriendo o explicando sus distintas partes, cualidades o circunstancias"; y, 3. "Definir imperfectamente algo, no por sus predicados esenciales, sino dando una idea general de sus partes o propiedades". Por su parte, para el verbo 'interpretar', algunas de sus acepciones son: 1. "Explicar o declarar el sentido de algo, y principalmente el de un texto"; 3. "Explicar acciones, dichos o sucesos que pueden ser entendidos de diferentes modos"; 4. "Concebir, ordenar 0 expresar de un modo personal la realidad" (se omiten las acepciones 2 y 5 a 7 ).

Con respecto a describir podemos retener lo siguiente: se da prelación a la representación, esto es, "al hacer presente algo con palabras o figuras que la imaginación retiene" (DRAE); esta presencia se hace a través del lenguaje (gramatical o pictórico) y tiene como fin mostrar cómo es algo. Ahora bien, la tercera acepción agrega una palabra aparentemente problemática: 'imperfectamente'. De acuerdo con el modo clásico de definir, una definición es perfecta siempre y cuando dé cuenta de la esencia de la cosa; la imperfección de la descripción radica en que no da cuenta de esto sino de sus cualidades in-esenciales. Por otra parte, en cuanto a la interpretación, la prelación la tiene la palabra 'explicar', bien sea el sentido o las acciones polisémicas, o que pueden ser comprendidas de múltiples modos; sin embargo, es destacable que también se agrega una nota problemática: la subjetividad. Mientras que en la descripción pareciera primar el respeto por mostrar cómo es el objeto, en la interpretación se da mayor énfasis al "modo personal", a lo subjetivo.

De acuerdo con lo anterior, descripción e interpretación son dos procesos diferentes: en el primero un sujeto usa su lenguaje (entendido más allá que simple gramática) para mostrar a otro cómo es un objeto. El éxito de la descripción estaría en que lo descrito correspondiera de modo fiel ("cabal": "completo, exacto, perfecto" (DRAE)) con el objeto, lo que presupone que el objeto es transparente a quien describe y a quien se describe. En el segundo, se parte de la premisa que el objeto a interpretar tiene un sentido ambiguo y que exige procesos reflexivos por parte del intérprete de modo que garantice que el sentido que propone para explicar el objeto ("algo") es viable o mejor que otros. En el primer proceso, la acción preponderante sería la observación; en el segundo, la capacidad que tiene el sujeto para establecer conjeturas acerca de un fenómeno. Para abreviar, en un tono popperiano, diríamos que la teoría (como conjetura) es lo que prima en la interpretación. Aclaramos que tomamos solamente el concepto popperiano de conjetura para desmarcar el concepto de teoría de su connotación positivista; en tal sentido, asumimos que las teorías científicas "no son una recopilación de observaciones, sino que son invenciones, conjeturas audazmente formuladas para su ensayo y que deben ser eliminadas si entran en conflicto con observaciones; observaciones, además, que raramente sean accidentales, sino que se las emprenda, como norma, con la definida intención de someter a prueba una teoría para obtener, si es posible, una refutación definida" (Popper 1994:72). La relación descripción-interpretación que defendemos en este escrito guarda estrecha relación con la relación observación-teoría (conjetura) planteada por Popper.

La historia del pensamiento ha mostrado que ambos procesos se han distribuido de modo preciso en ciertas épocas. Por ejemplo: se creyó que la observación-descripción era la forma más precisa como el 
científico natural daba cuenta de la naturaleza que investigaba, evitando que se "filtraran" interpretaciones carentes de sentido que pudieran alterar la representación "cabal" del mundo físico. Un caso paradigmático de esto lo constituyó el planteamiento acerca de las "proposiciones de observación" o "proposiciones protocolares", tan afín al positivismo lógico. Para Carnap, por ejemplo, el significado de una proposición se establece si al retrotraerse cada uno de sus términos se encuentra con una proposición elemental. Al respecto afirma: "En el caso de muchas palabras, específicamente en el de la mayoría de las palabras de la ciencia, es posible precisar su significado retrotrayéndolas a otras palabras ('constitución', definición). Por ejemplo: 'artrópodos son animales que poseen un cuerpo segmentado con extremidades articuladas y una cubierta de quitina'. De esta manera ha quedado resuelto el problema antes mencionado en relación a la forma proposicional elemental de la palabra 'artrópodo', esto es, para la forma proposicional 'la cosa $X$ es un artrópodo'. Se ha estipulado que una proposición de esta forma debe ser derivable de premisas de la forma ' $X$ es un animal', ' $X$ posee un cuerpo segmentado', ' $X$ posee extremidades articuladas', ' $X$ tiene una cubierta de quitina' y que inversamente, cada una de estas proposiciones debe ser derivable de aquella proposición" (Carnap 1965:68).

De otro lado, se creyó que, a diferencia de los fenómenos naturales, los fenómenos humanos y sociales no eran susceptibles de descripción, sino que requerían otro modo de aproximación que respetara el carácter cambiante, profundo, libre, propio de estos fenómenos. Empieza a considerarse, entonces, que los métodos de las ciencias naturales, donde se daba primacía a la observación y se pretendía el acceso a una realidad unificada, la misma para todos, no pueden aplicarse a estos fenómenos humanos y sociales, recurriéndose a la interpretación como forma de aproximación a ellos. Aunque esto ha sido ampliamente abordado (recuérdese que se dijo en la introducción que esta fue una polémica que ocupó a varios académicos en el siglo pasado pero que parece que ya se ha apaciguado), baste citar a uno de los más reconocidos filósofos de la interpretación o hermeneutas: "El concepto moderno de ciencia aparece marcado por el desarrollo de la ciencia natural del siglo XVII. A él debemos el creciente dominio de la naturaleza, y cabe esperar que la ciencia del hombre y de la sociedad nos permita alcanzar un dominio similar del mundo humano-histórico. Pero se espera más de las ciencias del espíritu al ver que el creciente dominio de la naturaleza, como producto de la ciencia, acrecienta el malestar en la cultura en lugar de reducirlo [...] Lo «científico» es aquí justamente destruir la quimera de una verdad desligada del punto de vista del sujeto cognoscente. Es el signo de nuestra finitud, que conviene no olvidar para tener a raya la ilusión [...] El conocimiento propio de las ciencias del espíritu implica siempre el autoconocimiento. Nada hay tan proclive al engaño como el autoconocimiento, pero nada tan importante, cuando se logra, para el ser del hombre" (Gadamer 2002: 43 y 46).

Se tiene, por tanto, que hubo un tiempo en el que la observación y su resultado, la descripción, estaban relacionadas de manera intrínseca con la ciencia natural, mientras que la interpretación se alojaba cómodamente en las ciencias humanas y sociales. Cada proceso garantizaba -o pretendía garantizar- la verdad (o "la verdad" dirían ciertos humanistas y científicos sociales) de sus afirmaciones. No obstante, algunas investigaciones cuestionaron la pretendida objetividad de las descripciones que se fundaban en la observación, denunciando que en tales descripciones había una importante carga de interpretación (Aguirre y Jaramillo 2013). Este hecho sirvió de aliciente a varias posturas filosóficas que concluyeron que si la propia investigación científico natural está cargada de teoría, es decir, carece de la objetividad que enorgullecía a los filósofos de la ciencia pertenecientes a la llamada concepción heredada, es preciso asumir la imposibilidad de una observación neutral; por ende, tampoco es posible una descripción "complet[a], exacta, perfecta" de los fenómenos naturales y, mucho menos, de los fenómenos humanos y sociales. En consecuencia, la aparente paz entre descripción e interpretación, entre observación y teoría, 
se rompe, concluyendo que toda observación está cargada de teoría (Hanson 1977) y que es absurdo buscar la objetividad (un testamento de esta postura es la compilación hecha por Watzlawick y Krieg 1995).

Podría decirse que en gran parte de las humanidades y de las ciencias sociales la discusión se inclinó a favor de la interpretación, descuidando, en cierto modo, la consideración mesurada del asunto de la observación, reducido peyorativamente al ámbito "positivista". Esto ha conducido a teorías que, un tanto acríticamente, adoptan una postura en contra del realismo directo, es decir, contra la posibilidad de tener "acceso perceptual al mundo real" (realismo) y que tal acceso pueda lograrse "sin necesidad de percibir algo más por medio de lo cual percibir el mundo real" (directo) (Searle 2015:15). Si bien es cierto que el ideal positivista de la observación neutra, condensada en enunciados protocolares, esto es, donde se reduce el papel del observador a su mínima expresión, era ingenua al no considerar los problemas propios que implica la observación humana, de ahí no se sigue que haya que dar el salto a una interpretación que vaya en contravía del realismo directo. En otras palabras, se podrá concluir que la observación more positivista estaba sostenida por una pobre teoría de la percepción; por tanto, es necesario que se emprendan estudios acerca del modo cómo los humanos percibimos la realidad que nos circunda sin comprometernos a ultranza con el subjetivismo o sin, necesariamente, rechazar de manera absoluta la objetividad.

En el caso específico de la investigación cualitativa, puede verse este descuido de la descripción y el aparente triunfo de la interpretación en un breve recorrido por sus manuales metodológicos. Tomemos los siguientes casos.

Caso 1. En la introducción a su clásico libro Qualitative research. Theory, method and practice, David Silverman presenta dos estudios agrupados bajo el título "Observación" (capítulo 2: "Etnografía: relacionando la parte y el todo" y capítulo 3: "Tendiendo puentes: la posibilidad del diálogo analítico entre etnografía, análisis de la conversación (o conversacional) y Foucault"). Allí afirma: "los dos capítulos sobre métodos observacionales buscan rescatar el trabajo observacional de las trampas (pitfalls) de la simple descripción y la perezosa codificación para dirigirse hacia metodologías y análisis excitantes en la investigación observacional" (2004:3, el énfasis es nuestro).

Caso 2. Definición del Diccionario SAGE de los métodos de investigación social: "Investigación que indaga sobre aspectos de la vida social, no susceptibles de medición cuantitativa. Asociada con una variedad de perspectivas teóricas, la investigación cualitativa usa un número de métodos que se centran en los sentidos y la interpretación de los fenómenos sociales y los procesos sociales en los contextos particulares en los que ocurren" (Sumner 2006:249, el énfasis es nuestro).

Los casos podrían multiplicarse ad nauseam; sin embargo, baste concluir este apartado con lo siguiente: en el debate describir-interpretar, la investigación cualitativa parece inclinarse hacia la interpretación en desmedro de la descripción, a la cual se le da un papel ínfimo. La descripción, a lo sumo, podría circunscribirse al ámbito de las ciencias naturales. En las humanidades y las ciencias sociales la descripción impregnaría a la investigación cualitativa de positivismo, algo que sugeriría obsolescencia. Ante esto planteamos que, si bien la descripción tal y como la propusieron los positivistas lógicos descuidó aspectos básicos de la observación y del observador al no tener en cuenta una sólida teoría de la percepción, esta no debe ser desatendida en la investigación cualitativa; es decir, la descripción cumple un papel preponderante en los procesos comprensivos. El siguiente apartado tratará de reivindicar el rol de la descripción en la investigación cualitativa, así como presentar una perspectiva de interpretación que dé importancia relevante a la observación. 


\section{Describir en investigación cualitativa: un verbo con derecho propio}

Este apartado está constituido por dos propuestas: en primer lugar, bosquejará las tesis básicas de la "Investigación Descriptiva" (descriptive research); en segundo lugar, expondrá, grosso modo, el planteamiento de la interpretación realista de Maurizio Ferraris, resaltando específicamente el lugar de la observación en esta versión de la interpretación. El objetivo, reiteramos, es darle el "derecho propio" a la descripción en la investigación cualitativa, tratando de centrar la atención en este proceso.

En el artículo "Whatever happended to qualitative description?", Margarete Sandelowski, desde la investigación en enfermería, propone "resucitar" un método para los investigadores cualitativos: la descripción cualitativa, de acuerdo con la autora, podría parecer "menos sexy" si se le compara con el incremento de métodos sofisticados teórica y técnicamente; por ello, sostiene que "es precisamente la creciente complejidad de los métodos cualitativos y de la tiranía del método en investigación en enfermería que se hace necesario el redescubrimiento de la descripción cualitativa" (2000:334). Sandelowski es consciente del lugar marginal que se le ha dado a la descripción, incluso, desde la investigación cuantitativa: "La investigación descriptiva se muestra en los libros de texto como perteneciendo al nivel más bajo en la jerarquía de la investigación cuantitativa. En esta jerarquía, los experimentos 'verdaderos' dirigidos a predecir y controlar son la regla de oro y cualquier otro diseño es no-experimental y débil” (2000:334). Según Sandelowski, esta visión de la descripción, en el contexto de la investigación cuantitativa, ha influenciado negativamente el modo como los investigadores cualitativos toman la descripción; al respecto afirma: "Muchos se han sentido obligados a defender sus esfuerzos como algo más que mera descripción; es decir, han buscado 'credibilidad epistemológica' al diseñar su trabajo como fenomenología, teoría fundamentada, etnografía o estudios narrativos. En muchos casos, sin embargo, este esfuerzo ha resultado en 'posturas' acerca de la fenomenología, la teoría fundada, la etnografía o los estudios narrativos, más que en fenomenologías, teorías, etnografías o interpretaciones narrativas" (2000:334). Esto ha llevado a que, por ejemplo, se tome como fenomenológica una investigación que "puede que no incluya más que reportes de las experiencias 'subjetivas' de los participantes" (2000:334).

De acuerdo con lo anterior, Sandelowski encuentra un vacío teórico acerca de lo que significa la descripción cualitativa como método distinto pero de igual peso que los otros métodos cualitativos, pese a que es uno de los enfoques metodológicos empleados con más frecuencia en las disciplinas prácticas. Para subsanar esto, la autora parte de tres supuestos: 1. La descripción cualitativa es una alternativa "categórica" de investigación, esto es, el método ya existe, solo que es desconocido; en esto se opone a las alternativas "no categóricas" o adaptaciones de la fenomenología, teoría fundamentada y etnografía a la investigación en enfermería; 2 . Los estudios descriptivos cualitativos son menos interpretativos que la "descripción interpretativa", esto es, en aquellos no se requiere que los investigadores se muevan de los datos; 3. Los investigadores no requieren un tratamiento conceptual u otro modo abstracto para abordar los datos.

La tesis que defiende Sandelowski es la siguiente: si bien es cierto que ninguna descripción está libre de interpretación, la descripción cualitativa básica o fundamental implica un tipo de interpretación que es de baja inferencia (low-inference) o, probablemente, conduce a un fácil consenso entre los investigadores. Esta tesis la ilustra con el siguiente ejemplo: "Incluso aunque un investigador pueda representar los sentimientos y otro investigador los sucesos reportados por una mujer en una entrevista, ambos investigadores estarán probablemente de acuerdo en que, por ejemplo, la mujer dijo varias veces que estaba enojada y que dijo que su madre murió un día después de que supo que tenía cáncer de seno. En 
el caso de los dos investigadores que describen ostensiblemente la misma escena, un investigador podría mostrar la disposición espacial en la habitación, mientras el otro investigador mostrará las interacciones sociales. Cada investigador debería estar de acuerdo con las descripciones del otro como reproducciones exactas de la escena. Es así como, con las descripciones de baja inferencia, los investigadores estarían de acuerdo más rápidamente sobre los 'hechos' del caso, incluso si no presentan los mismos hechos en sus descripciones" (Sandelowski 2000:335).

Infortunadamente, la propia Sandelowski, al utilizar las comillas para la palabra hechos, pareciera estar dándole lugar a quienes niegan los hechos (por ejemplo, los constructivistas) y, de paso, debilitando su propuesta. No obstante, la explicitación de su tesis necesariamente se compromete con la visión realista (esto es: hay realidad extra-mental), la cual le permite afirmar que: "Las descripciones siempre deben transmitir de manera precisa los eventos en su propia secuencia, o tener validez descriptiva, y los significados que los participantes atribuyen a aquellos eventos, o tener validez interpretativa" (2000:336). Según esto, tanto en la descripción como en la interpretación debe haber un criterio de validez que impida el simple juego de opiniones o la libre asociación de ideas. El criterio parte de la fidelidad al hecho, lo que garantizaría su estatus de hecho (ontología) y la posibilidad de acceder a él de modo válido (epistemología) a través de un proceso que exige rigor (metodología).

Es claro que Sandelowski no pretende derrocar ninguno de los métodos cualitativos presentes en las humanidades y las ciencias sociales, sino "resucitar" o reivindicar el papel de la descripción cualitativa en la investigación social. ¿Cuál sería su lugar? ¿Cuál sería su papel? En la conclusión de su artículo afirma: "El estudio descriptivo cualitativo es el método que se puede elegir cuando se deseen descripciones rigurosas de los fenómenos. Tal estudio es especialmente útil para investigadores que busquen saber el quién, qué y dónde de los eventos. Aunque fundamental a todo enfoque cualitativo de investigación, los estudios descriptivos cualitativos comprenden una valiosa aproximación metodológica en y por sí mismos. Los investigadores pueden, sin sonrojarse, nombrar su método como descripción cualitativa" (2000:339).

Esta primera propuesta plantea posibilidades para la investigación cualitativa y, desde una perspectiva epistemológica, reivindica el papel de la observación-descripción en humanidades y ciencias sociales. A partir de la categoría "baja inferencia", la autora reconoce que si bien "la descripción cualitativa básica es inevitablemente interpretativa, en tanto está "filtrada a través de las percepciones (humanas)»" (2000:336), es posible una presentación de los hechos, en lenguaje cotidiano, susceptible de validación intersubjetiva. Ahora bien, la indagación de nuestro artículo es acerca del papel de la descripción en la investigación cualitativa; de acuerdo con la propuesta de Sandelowski, la descripción es protagónica a la hora de responder preguntas cualitativas con un interés específico, sin necesidad de adornarla con otros enfoques; además, ocupa un lugar definido en cada uno de los métodos cualitativos y podría decirse que su papel consiste en la preservación de los hechos, precaución para no hacer de ellos una ficción.

La segunda propuesta a considerar es la del profesor italiano Maurizio Ferraris denominada "Nuevo Realismo", que consiste en plantear un "nuevo enfoque realista a la hermenéutica" (nuovorealismo.wordpress.com). Por sí misma -y por las discusiones que ha generado-, esta propuesta requeriría un estudio amplio; sin embargo, de acuerdo con los intereses de este artículo, nos proponemos identificar solo aquellos elementos que nos permiten considerar el lugar de la descripción en la investigación cualitativa. Esta discusión es importante, máxime si se recuerda que se había planteado que la investigación cualitativa es por excelencia interpretativa, hermenéutica, lo cual incluye una alta dosis de subjetivismo y, en consecuencia, parecería que queda en entredicho la objetividad. De modo contrario, 
Ferraris respalda la posibilidad de conservar la objetividad en ciencias humanas y sociales, no obstante su carácter interpretativo.

En su artículo "On New Realism", Ferraris afirma: "Los 'nuevos realistas' provienen de la filosofía continental donde el peso del antirrealismo fue mucho mayor que en la filosofía analítica. Ambas tradiciones tienen en común una premisa: no hay 'cosas en si', sino solo fenómenos mediados (o creados) por nuestros esquemas conceptuales y nuestro aparato perceptual” (2013:45). De acuerdo con esto, la tradición continental que propone y defiende el antirrealismo niega el realismo directo a la luz de dos tesis centrales: el esquema conceptual ("primacía del concepto en la construcción de la experiencia") y las particularidades de la percepción, específicamente, la imposibilidad de acceder a la realidad tal como es, es decir, a los hechos desnudos; a lo sumo, podemos acceder a lo extra-mental en los límites que nuestro aparato perceptual lo permite, mas no a la cosa misma.

En el asunto puntual de la observación, Ferraris sostiene: "El que la experiencia perceptual dependa de la explicación conceptual nos lleva a caer en lo que los psicólogos llaman: 'stimulus error', que se refiere a la naturalidad con la que tendemos a sustituir una observación con una explicación; es la facilidad con la que, cuando nuestros ojos están cerrados, respondemos 'nada' o 'negro', cuando alguien nos pregunta: '¿qué ves?', cuando en realidad estamos viendo fosfenos y brillos, que no explicamos desde un nivel descriptivo, porque a lo que nos referimos es a algo diferente: una teoría de la visión según la cual el ojo es como una cámara oscura, en la que, cuando el diafragma está cerrado, solo hay obscuridad absoluta. Cuando decimos que los observadores equipados con teorías diferentes ven la realidad en cuestión de modo distinto, se confiere una dignidad filosófica a un error psicológico; de modo más relevante, se comete un error categorial al confundir, en este caso particular, ver con conocer" (2013:50).

De acuerdo con esta cita, el desprecio por la observación, en aras de la interpretación, puede estar basado en un error explicado por la psicología, error que, como muchos, ha alimentado diversas posturas filosóficas. La propuesta realista de Ferraris está en abierta confrontación con el antirrealismo y una de sus figuras más populares, el construccionismo, que si bien tienen una larga tradición, brotaron con fuerza gracias al impulso del postmodernismo, incrustándose en los discursos de las ciencias humanas y sociales, en general, y en las metodologías de tales ciencias, en particular. Por tanto, aunque Ferraris se propone establecer una "paz perpetua" entre realistas y antirrealistas, dándole razón a cada bando, pueden leerse sus aportes como una clarificación de erróneos presupuestos que afectan a la interpretación.

La simple denuncia de error, otorgada por el stimuli error, parece echar por tierra los presupuestos antirrealistas: 1. Trascendentalismo ("Lo que hay (ontología) está determinado por lo que conocemos (epistemología)"); 2. Pragmatismo ("Nada trasciende los esquemas conceptuales con los cuales identificamos nuestras prácticas cotidianas"); 3. Postmodernismo ("Estos esquemas están determinados, a la vez, por otros esquemas") (Ferraris 2014:76). No obstante, Ferraris se empeña en mostrar cómo todo esto parte de la confusión entre ontología y epistemología, confusión que agrupa bajo la llamada "falacia trascendental". No reconstruiremos punto por punto tal falacia, ni los argumentos que propone Ferraris para refutarla; basta recoger algunos elementos para fortalecer la idea que defendemos.

En primera instancia, Ferraris afirma: "«Es prudente no fiarse nunca por entero de quienes nos han engañado una vez». Así, en la página inicial de las Meditaciones, Descartes propone enseñarnos a no confiar en los sentidos, aquellos siervos indignos que, en su opinión, nos han engañado y, en consecuencia, haríamos bien en desconfiar sistemáticamente. De forma consistente con este punto de partida, Descartes 
sostiene que la certeza no debe buscarse por fuera, en un mundo repleto de errores sensibles, sino adentro, en el cogito, el asiento de las ideas claras y distintas" (2014:76).

Ferraris enuncia la consecuencia de esto del siguiente modo: "la falacia socava la certeza primitiva y areflexiva con la que nos relacionamos con el mundo [...] pero no ofrece alguna otra certeza que la reemplace. La incerteza acerca del conocimiento -la desconfianza total en los sentidos y la inducción- es concebida como si llevara una carga de consecuencias ontológicas, como si fuera capaz de desmantelar las estructuras de la realidad" (2014:77). De acuerdo con esto, la observación-descripción queda relegada como fuente de conocimiento, siendo relevante el papel del sujeto cognoscente. Los construccionistas radicalizaron esta postura concluyendo que lo que vemos depende de lo que conocemos.

En segunda instancia, del hecho que interactuamos con el mundo por medio de esquemas conceptuales, no se sigue que nuestros esquemas conceptuales determinen el mundo. Ferraris lo ilustra de este modo: "Puedo conocer que hay agua en el vaso y que su fórmula química es $\mathrm{H}_{2} \mathrm{O}$, o puedo no saber eso; pero las propiedades del agua permanecen iguales. Es crucial no confundir la epistemología con la ontología (2014:81). Este punto, tomado de Searle, es muy importante en la discusión descripción-interpretación. Más adelante se ubicará en este contexto.

Finalmente, en el apartado "Mundo interno y mundo externo", Ferraris establece un conjunto de consecuencias que se derivan de su consideración acerca del carácter no-enmendable de la realidad: "La no enmendabilidad nos dice algo acerca de la existencia de un mundo que es externo no solo a nuestros cuerpos [...] sino también a nuestras mentes" (2014: 83). De estas consecuencias nos interesa resaltar la autonomía del mundo con relación a los esquemas conceptuales y perceptuales: "La realidad posee una conexión estructural y estructurada que no solo resiste los esquemas conceptuales y perceptuales, estableciendo así la no enmendabilidad, sino que también los precede [...] Tal mundo existe, de otra manera, no seríamos capaces de distinguir el conocimiento del sueño" (2014:85).

Lo planteado por Ferraris podría entenderse como una visión más acerca del debate entre realistas y antirrealistas, tan familiar a la filosofía analítica de la ciencia. En cierto modo, ninguno de los argumentos fuertes de Ferraris aporta algo nuevo al debate. No obstante, el que sea un hermeneuta -y no un filósofo de la ciencia-, cercano a Derrida o Husserl, formado en la tradición continental, implica que la hermenéutica como disciplina requiere un examen riguroso a la luz de las tesis realistas. Las tesis de Ferraris reivindican, frente a la hegemonía de la interpretación, la existencia del hecho, y no solo esto, también la posibilidad de acceder a él de modo objetivo. En consecuencia, la pregunta que motiva este artículo podría aprovechar las tesis de Ferraris de la siguiente manera: 1. Debilitando el privilegio de la interpretación al rescatar que hay hechos; 2. Buscando un encuentro entre hechos (intuiciones) e interpretaciones (conceptos); 3. Evaluando en su justa medida el dicho kantiano, quien, junto con Descartes, sustenta la falacia trascendental al sostener que "las intuiciones sin conceptos son ciegas". Completando la otra cara, extrañamente obviada por Ferraris, "los conceptos sin intuiciones son vacíos" (Kant 2009:100).

En cuanto a la descripción, pese a no ser abordada por Ferraris de modo directo, podría decirse que garantizando la existencia del mundo externo, extra-mental, independiente de la mente, es decir, argumentando a favor de la realidad no enmendable, se abre la posibilidad de describirla de modo tal que coincida con el estado de hechos. Por tanto, la descripción valdría por sí misma en tanto refleja el qué y el cómo de la realidad, o serviría de criterio para determinar hasta qué punto se sostiene una interpretación en vez de otra; esto es, salvaguardando la objetividad en ciencias humanas y sociales. 
Creemos que las propuestas consideradas en este apartado, junto con las reflexiones iniciales acerca del asunto, nos permiten responder, de forma puntual, a la pregunta del artículo. En este sentido, el siguiente apartado conclusivo reunirá, bajo la lente de la investigación cualitativa, las piezas hasta ahora dispersas.

\section{La descripción: garante de validez y preservación del otro en la investigación cualitativa}

Este último apartado intenta brindar orientaciones prácticas que algunos etnógrafos e investigadores cualitativos encuentran en la descripción; si bien se pueden hallar bastantes beneficios en torno al método descriptivo, queremos enunciar al menos tres razones del por qué y cómo hacer uso de la descripción en nuestras investigaciones: la primera en relación a su permanencia en los diseños cualitativos a pesar de las modas posmodernistas y posestructuralistas que han sobrevenido a la investigación cualitativa; la segunda, en relación a cómo la descripción protege, de cierto modo, la alteridad del otro, en tanto es un intento por registrar sus comportamientos e interacciones más allá de nuestros esquemas mentales; la tercera, en relación a considerar la descripción como garante de validez en el proceso de registro y análisis de los datos. Consideramos que estas razones justifican, al menos en parte, la rigurosidad que no debe faltar en los diseños cualitativos, por fenomenológico, etnográfico, de teoría fundamentada o etnometodológico que este sea.

La antropóloga Jean Langdon en su libro La negociación de lo oculto, confiesa cuatro décadas después de haber realizado su tesis doctoral, que experimentó un cambio radical en el campo académico de la antropología: "sus discursos, conceptos y preocupaciones son totalmente diferentes a aquellos de 1970", continúa: "Como antropólogos, se nos dirigen preguntas completamente diferentes sobre la realidad social. El campo ha sido objeto de una reflexión crítica sobre sus conceptos centrales, sus premisas epistemológicas y las formas como construyen el conocimiento. En vez de presentar las etnografías como descripciones objetivas e integrales de pueblos sin historia, ahora reconocemos la agencia del otro, nuestra subjetividad, la naturaleza fragmentada de nuestras etnografías y las limitaciones de nuestras interpretaciones" (2014:11).

Parecería que las "descripciones objetivas" hicieron parte de un trabajo de campo externo al investigador, riguroso, elaboradas según principios establecidos por aquellos primeros etnógrafos como Malinowski: "observación participante, estadía de mucho tiempo, aislamiento geográfico y social, aprendizaje del idioma y registro de la experiencia en diarios de campo" (Langdon 2014:11). Tanto en la antropología, como en la investigación cualitativa en general, se crean desplazamientos que van haciendo del trabajo de campo un asunto más protagónico por parte del investigador; cambios donde se privilegia más la interpretación que la laboriosidad descriptiva que se pueda alcanzar al interior del mismo proceso; se entra, de este modo, a rupturas y "modas" en los diseños cualitativos, donde la descripción pasó a ser un modo positivista de obtener datos a la antigua por parte de etnógrafos "de terreno", que ejercían su labor desde una etnografía eminentemente clásica.

Yvonna Lincoln y Norman Denzin identificaron un quinto momento o periodo histórico de la investigación cualitativa, como una tendencia que tiende a generar desplazamientos de una moda intelectual a otra: "desde el positivismo al postpositivismo, la semiótica y el estructuralismo, el postestructuralismo y el postmodernismo, y así sucesivamente" (1994:576); fruto de esto, los autores consideran que la "historia de la investigación cualitativa se define más por cortes y rupturas que por una clara evolución o un movimiento progresivo desde una etapa a la siguiente. Estos cortes y rupturas se mueven en ciclos y fases, por eso lo que hoy es algo pasado de moda quizá dentro de una década esté en boga (1994:576). Por ello, citando a Bruner, exponen que se hace necesario ir a las fuentes de los textos "fuera de moda", 
básicamente por dos razones: la primera, para reaprender de las primeras etnografías -caso de la tesis de Jean Langdon- con el fin de ver si el criticismo clásico aún se mantiene; la segunda, para estudiar los trabajos de estas tradiciones y así comprender cómo escribieron estos maestros en una época considerada ya "pasada de moda". Lincoln y Denzin concluyen que "aunque estemos seguros de que los presupuestos postpositivistas del abordaje de la teoría general deban ser criticados, el método general para construir interpretaciones a base de observaciones e interacciones con el mundo no se va a modificar" (1994:578). En tal sentido, la base sólida de una interpretación es la observación y su posterior descripción; esta debe mantenerse más allá de los modismos, ambages y giros posmodernos que van cambiando lo que actualmente se entiende por investigación cualitativa. Lincoln y Denzin enfatizan que el compromiso de los investigadores cualitativos siempre será estudiar la experiencia humana desde los mismos significados de los participantes, cómo ellos "hacen y viven historias que han sido arrancadas de las manos de los fantasmas del pasado" (1994:17).

Ahora bien, parece ser que estas modas tienen su origen cuando se cuestiona el papel que cumple el investigador, como observador, al momento de consignar sus descripciones; es decir, qué tanto interviene su self o yo-investigador en lo observado, a su vez, cómo observar a otros que también lo observan a él; siendo así, como investigador le es imposible desprenderse de una subjetividad que le lleva a registrar su participación en el proceso descriptivo; podría decirse que ya hay, de antemano, un papel interpretativo por parte del observador frente a: la manera de entrar en relación con los participantes, su posición en el campo y cómo registra su intervención en las notas de campo; por tanto, existe una pre-comprensión o intencionalidad inicial al momento de observar y registrar los datos, lo cual no es desconocido al interior de la misma descripción. Harry Wolcott, de hecho, considera que existe una subjetividad frente a lo que se observa y registra en las notas de campo: "no pretendas estar por encima del hecho de que no existe una descripción pura... Si no fuésemos selectivos, y por ende subjetivos, en nuestro enfoque, no seríamos capaces de construir narración alguna. Sin algunas ideas preconcebidas de lo que se va a describir, no puede haber descripción. Cada paso del camino refleja procesos conscientes e inconscientes de enfoque y selección" (2003:41).

No obstante, el ser conscientes de nuestra influencia en el campo y, por ende, de nuestras descripciones, nos posibilita hacer uso de una reflexividad que nos permite ser más críticos frente a lo que debemos registrar o no; es decir, qué tanto de lo que consignamos es producto de nuestras representaciones mentales y qué tanto es de los participantes; la reflexividad nos desnuda de una conciencia aprehensiva respecto a cómo estamos comprendiendo al otro investigado, otro que sobrepasa nuestras intencionalidades y juicios de valor. De todas maneras, si bien el proceso de observación está impregnado de una subjetividad que nos lleva a ser conscientes del modo como estamos registrando los datos, este no un es argumento suficiente para considerar que todo lo descrito es de orden interpretativo; no en vano, Sandelowski considera que es posible hablar de una interpretación de baja inferencia, es decir, más pegada a los datos y desapegada a nuestro propio yo. "Digan lo que digan los posmodernos, sí existe el mundo exterior", nos dice Wolcott (2003:37); de manera sencilla, el autor define la descripción "como la fundación sobre la cual se construye la investigación cualitativa. A menos que demuestres ser un talentoso conceptualizador o intérprete, la descripción probablemente constituirá la contribución más importante que hagas. Mientras más sólida sea la base descriptiva, es probable que sobreviva mejor a las volubles modas sobre presentación de informes o al cambio de énfasis en la forma de derivar significado de nuestros estudios. Dale a tu relato una base firme en la descripción" (2003:35).

Toda investigación se basa en unos datos de observación; las contribuciones genuinas que hagamos a partir de nuestros trabajos estarán fundadas en la solidez de nuestras descripciones. Incluso Wolcott llega 
a recomendar, al menos en los inicios del proceso de escritura cualitativa, que se resista a "la tentación de interrumpir el trabajo con excursiones prematuras al análisis o interpretación, excepto para señalar asuntos a los que se tiene pensado regresar. Esto permitirá que el informe final goce de bases firmes para saber hasta qué punto es importante sustentar y comparar las descripciones con trabajos ajenos" (2003:41); la descripción es la que posteriormente nos anima a buscar el tipo y número de fuentes teóricas para iniciar con el proceso interpretativo y no considerar, por el contrario, que son las fuentes teóricas las que iluminan y dan vida a los datos descriptivos.

En relación a la alteridad, una cuidadosa descripción posibilita que se dé un registro, lo más próximo posible, del otro investigado o participante. El otro se escapa de nuestros esquemas mentales para increparnos que él es mucho más de lo que podamos registrar acerca de él; aun así, es más seguro una descripción esmerada de sus comportamientos e interacciones, que una interpretación fundada en nuestra propia manera de ver y concebir su mundo social. Además, se ha vuelto "normal" que los informes cualitativos terminen relatando más las vivencias, emociones y sentimientos del investigador en el campo, que la vida misma de los participantes; Lincoln y Denzin, como un modismo más, afirman que cada vez "nos preocupamos menos de nuestra 'objetividad' como cientistas que por suministrarles a nuestros lectores en nuestros textos alguna experiencia del Otro que sea lo más poderosa posible desde el punto de vista proposicional, tácito, intuitivo, emocional, histórico, poético e incluso enfático" (1994:583). Los autores recurren nuevamente a Bruner para advertir que "el peligro es poner el yo personal tan profundamente detrás y dentro del texto que termina dominándolo completamente. Entonces el trabajo deviene narcisista y egotista. Nadie está llamando a la auto-compasión etnográfica" (1994:584). Pues bien, la descripción hace que no nos desenfoquemos del significado que nos ofrecen los otros frente al tema y objeto de estudio que tratamos en la investigación; por comprometida que sea nuestra participación en el campo, se debe tener claro que la subjetividad del investigador en el campo es evidente pero no protagónica; es real, pero discreta; por ello, "la primera tarea que se impone en la elaboración de la descripción es una lectura minuciosa y rigurosa de los registros, es decir, una lectura en la escucha... en los registros es donde encontramos al otro, su voz, sus visiones del mundo que lo rodea y de sus relaciones con los otros" (De Tezanos 2002:147).

Por otra parte, al reconocer las múltiples voces de los participantes en el estudio, es importante describir cómo estos van dando una organización a sus propias vivencias y comportamientos, esto antes de encuadrarlos en una lógica prediseñada guiada generalmente por un enfoque teórico. Con facilidad se cae en el error de hacer coincidir las experiencias de los participantes entre sí: bien sea por el uso del lenguaje en la similitud de las palabras, o porque varios participaron de una misma situación, o porque están en una misma categoría étnica o cultural; se olvida que los participantes, aparte de narrar su experiencia, otorgan un orden y una lógica que muchas veces, en su conjunto, no coincide con la lógica interpretativa del investigador. Pues bien, una buena descripción nos permite "ir encontrando el orden que los sujetos a quienes [se] observó y entrevistó, le dan a la realidad estudiada... de alguien que va dando forma a un conjunto de piezas fragmentadas, en cuyo interior se encuentran las representaciones de los sujetos y que solo adquieren significado cuando se las mira como una totalidad" (De Tezanos 2002:148).

Del mismo modo, Harry Wolcott advierte que tendemos a caer en la inferencia de lo que el participante nos dice, antes que observar lo que precisamente nos está diciendo; para él, existe una sutil diferencia entre datos observados y los inferidos: "solo desde tu propia perspectiva puedes informar sobre qué 'sentía' alguien con respecto a lo que estaba sucediendo, o sobre los 'significados' que le atribuían. A menos que otras personas expresen tales sentimientos, lo que debemos informar es lo que en realidad vimos y escuchamos, nunca lo que inferimos, a no ser que podamos sustentar nuestras observaciones", 
por ello, "la descripción cuidadosa exige un sentido de desapego" (2003:36); lo cual implica estar vigilantes a cualquier tentativa de asimilación que se haga del otro.

Finalmente, la descripción es también garante de validez frente a los datos obtenidos; como lo anunciaba Sandelowski, tanto en la descripción, como en la interpretación, debe haber un criterio de validez que nos guarde de caer en un mar de opiniones o de libre asociación de ideas. Tanto Wolcott, como De Tezanos, recomiendan iniciar con descripciones sencillas del entorno, de acontecimientos y hechos sucedidos al interior del escenario; estos datos se deben registrar de manera cuidadosa y con un adecuado nivel de detalle; de hecho, Wolcott advierte: "si solo tienes dentro de ti dos párrafos de discurso florido, guárdalos para otra ocasión y limítate a proseguir con el informe descriptivo sencillo" (2003:35). Son las descripciones sencillas y detalladas las que pasan a ser la base de interpretaciones que se soportan en observaciones cuidadosas y que hacen del investigador un ser que no pierde detalle, tanto de lo que acontece en el exterior, como de lo que le pasa en su interior.

Las especificaciones del contexto y de los participantes son evidencia de un mundo real y externo a nosotros, lo que implica ir al "terreno de campo" como un lugar que desborda nuestras construcciones mentales y sociales; la antropóloga Rosana Guber considera que las especificaciones detalladas del contexto son las que posibilitan que nos sorprendamos en el campo y a su vez sean el fundamento de la teorización: "la especificación no aporta a la explicación del nivel teórico, como se ha pretendido en numerosas investigaciones antropológicas; aporta sí la materia sobre la cual se estructurará la teorización, hacia una explicación que incluya a los actores en sus contextos específicos" (2004:288). Igualmente, De Tezanos expone que "el texto que surge del proceso descriptivo está recogido única y exclusivamente desde los registros. Lo único que ilumina la estructura y escritura de este texto es el objetivo general del proyecto" (2002:149).

En conclusión, la descripción es garantía de la validez del trabajo; esta nos lleva a distinguir un aceptable informe cualitativo de uno inaceptable, o una buena investigación cualitativa de una mala. Un buen informe se logra cuando el investigador ha realizado una correcta interpretación del significado de los participantes, diferenciando su propio significado como intérprete; además, "lo hace de manera explícita, fundamentada, en función de salvaguardar el sentido auténtico del texto... Esto sería algo así como el máximo límite de objetividad posible, dentro del nuevo encadenamiento o espiral de significados que se da en el método hermenéutico" (García 2014:102), lo que da a la hermenéutica un piso sustentable para la interpretación, así como a los demás métodos cualitativos al interior de las ciencias humanas y sociales.

\section{Agradecimientos}

Este artículo es parte de las discusiones adelantadas en el grupo de investigación Fenomenología y Ciencia (Categoría B - Colciencias). Los autores dan expreso crédito y mención a la Universidad del Cauca por los espacios y tiempos permitidos para el desarrollo de este trabajo.

\section{Bibliografía}

Aguirre, J. y Jaramillo, L. 2013. Tesis de la carga teórica de la observación y constructivismo. Cinta de Moebio 47: 74-82. doi: 10.4067/S0717-554X2013000200002

Carnap, R. 1965. La superación de la metafísica mediante el análisis lógico del lenguaje. En: A.J. Ayer. El positivismo lógico. México: Fondo de Cultura Económica. 
De Tezanos, A. 2002. Una etnografía de la etnografía. Bogotá: Antropos.

Ferraris, M. 2012. Manifesto del nuovo realismo. Roma: Laterza.

Ferraris, M. 2013. On new realism. Kairos. Revista de Filosofia \& Ciência 8: 45-65.

Ferraris, M. 2014. Un nuevo enfoque realista a la hermenéutica. En: J. Aguirre. Cuadernos de epistemología, número 6. Popayán: Universidad del Cauca, pp. 75-92.

Gadamer, H.G. 2002. Verdad y método II. Salamanca: Sígueme.

García, W. 2014. Acerca de la hermenéutica, la interpretación y la comprensión. En: J. Aguirre. Cuadernos de epistemología, número 6: reflexiones en torno a la filosofía de la ciencia y la epistemología. Popayán: Universidad del Cauca, pp. 93-106.

Giorgi, A. 2014. An affirmation of the phenomenological psychological descriptive method: a response to Rennie. Psychological Methods 19(4): 542-551. doi: 10.1037/met0000015

Guber, R. 2004. El salvaje metropolitano. Reconstrucción del conocimiento social en el trabajo de campo. Buenos Aires: Paidós.

Hanson, N. R. 1977. Patrones de descubrimiento: observación y explicación. Madrid: Alianza Editorial

Johnson, B; Onwuegbuzie, A. y Turner, L. 2007. Toward a definition of mixed methods research. Journal of Mixed Methods Research 1(2): 112-133. doi: 10.1177/1558689806298224

Kant, I. 2009. Crítica de la razón pura. México: Fondo de Cultura Económica.

Langdon, J. 2014. La negociación de lo oculto: chamanismo, medicina y familia entre los siona del bajo Putumayo. Popayán: Universidad del Cauca.

Lincoln, Y. S. y Denzin, N. K. 1994. Handbook of qualitative research. Thousand Oaks, CA: Sage.

Popper, K. 1994. Conjeturas y refutaciones. El desarrollo del conocimiento científico. Barcelona: Paidós.

Rennie, D.L. 2012. Qualitative research as methodical hermeneutics. Psychological Methods 17(3): 385398. doi: $10.1037 / \mathrm{a} 0029250$

Sandelowski, M. 2000. Whatever happened to qualitative description? Research in Nursing \& Health 23: 334-340. doi: 10.1002/1098-240X(200008)23:4<334::AID-NUR9>3.0.CO;2-G

Searle, J. 2015. Seeing things as they are. A theory of perception. Oxford: Oxford University Press.

Silverman, D. 2004. Qualitative research. Theory, method and practice. London: Sage. 
Sumner, M. 2006. Qualitative research. En: V. Jupp (Ed.). The Sage Dictionary of Social Research Methods. London: Sage.

Watzlawick, P. y Krieg, P. 1995. El ojo del observador. Barcelona: Gedisa.

Wolcott, H. 2003. Mejorar la escritura de la investigación cualitativa. Medellín: Universidad de Antioquia.

Recibido el 23 Abr 2015

Aceptado el 14 Jun 2015 